\title{
Impact of Signal-Conjugate Wavelength Shift on Optical Phase Conjugation-based Transmission of QAM Signals
}

Da Ros, Francesco; Lillieholm, Mads; Yankov, Metodi Plamenov; Guan, Pengyu; Hu, Hao; Forchhammer, Søren; Galili, Michael; Oxenløwe, Leif Katsuo

\section{Published in:}

Proceedings of ECOC 2017

Publication date:

2017

Document Version

Peer reviewed version

Link back to DTU Orbit

Citation (APA):

Da Ros, F., Lillieholm, M., Yankov, M. P., Guan, P., Hu, H., Forchhammer, S., Galili, M., \& Oxenløwe, L. K. (2017). Impact of Signal-Conjugate Wavelength Shift on Optical Phase Conjugation-based Transmission of QAM Signals. In Proceedings of ECOC 2017 IEEE.

\section{General rights}

Copyright and moral rights for the publications made accessible in the public portal are retained by the authors and/or other copyright owners and it is a condition of accessing publications that users recognise and abide by the legal requirements associated with these rights.

- Users may download and print one copy of any publication from the public portal for the purpose of private study or research.

- You may not further distribute the material or use it for any profit-making activity or commercial gain

- You may freely distribute the URL identifying the publication in the public portal 


\title{
Impact of Signal-Conjugate Wavelength Shift on Optical Phase Conjugation-based Transmission of QAM Signals
}

\author{
F. Da Ros, M. Lillieholm, M.P. Yankov, P. Guan, H. Hu, S. Forchhammer, M. Galili and L.K. Oxenløwe \\ (1) Department of Photonics Engineering, Technical University of Denmark, 2800 Kgs. Lyngby, \\ Denmark fdro@fotonik.dtu.dk
}

\begin{abstract}
The impact of signal-conjugate wavelength shift on nonlinearity compensation through optical phase conjugation is investigated for 64- and 256-QAM. Wavelength-shift independent achievable rate improvements between 0.2 and 0.3 bit/symbol are reported for shifts up to $30 \mathrm{~nm}$ in $500-\mathrm{km}$ transmission.
\end{abstract}

\section{Introduction}

Current optical transmission systems are limited in their transmission reach and rate by the Kerr nonlinearity in optical fibers, which sets an upper limit on the power that can be transmitted without significantly distorting the data signal. Several techniques are being investigated to provide compensation for the nonlinear distortion, both in the digital domain, e.g. digital backpropagation (DBP), and all-optically, e.g. through optical phase conjugation (OPC $)^{1-6}$. Compared to DBP, OPC enables better nonlinearity compensation for wavelength division multiplexing (WDM) systems without increasing the electrical bandwidth of the transceivers ${ }^{2}$. Ideal nonlinearity compensation through OPC requires symmetric power and dispersion profiles of the link with respect to the position of the OPC stage, as well as a link with no wavelength dependent dispersion and no polarization mode dispersion (PMD). The impact of link symmetry has been extensively investigated ${ }^{3}$, and the effects of PMD have been numerically analysed ${ }^{4}$. However, to the best of our knowledge no systematic investigation of the impact of wavelength dependent dispersion on OPC systems has been reported. Understanding the impact such wavelength dependency is critical, as the OPC is typically implemented using four-wave mixing (FWM), resulting in a wavelength shift between the signal and its conjugated copy. Even though schemes providing wavelength-shift free operation are being investigated ${ }^{6-8}$, the techniques reported so far are limited to single-channel operation and would yield a mirroring of a WDM channel band ${ }^{6}$.

In this work, we investigate the impact of signal-conjugate wavelength shift on the improvement provided by the OPC operation. We consider different shifts in a transmission system using 64- and 256-quadrature amplitude modulation (QAM) signals.

\section{Experimental setup}

The experimental setup is shown in Fig. 1. A 64or 256-QAM signal is generated from an external cavity laser (ECL, $10-\mathrm{kHz}$ linewidth) modulated in an IQ modulator driven by an arbitrary waveform generator (AWG, $64 \mathrm{GSa} / \mathrm{s}$ ) operating at $10 \mathrm{GBd}$ and $16 \mathrm{GBd}$. The QAM symbols are interleaved with quadrature phase shift keying (QPSK) pilots at a rate of $10 \%$, and pulse shaped with square root raised cosine (roll-off factor $=0.1$ ). The transmitter rate has been set to $4.5 \mathrm{bit} / \mathrm{QAM}$ symbol, and a split-and-combine polarization emulator (Pol. mux) generates a polarization division multiplexed (PDM) signal, resulting in rates of $81 \mathrm{~Gb} / \mathrm{s}$ and $130 \mathrm{~Gb} / \mathrm{s}$. The PDM signal is polarization scrambled and launched into the first transmission link. Each of the two links consists of three $84-\mathrm{km}$ spans of standard single mode fiber (SSMF), with erbium doped fiber amplifiers (EDFAs) compensating for the fiber loss. While such a link configuration is not optimum for nonlinearity compensation by OPC, due to the lack of symmetry, it does represent a configuration used in many deployed links. OPC

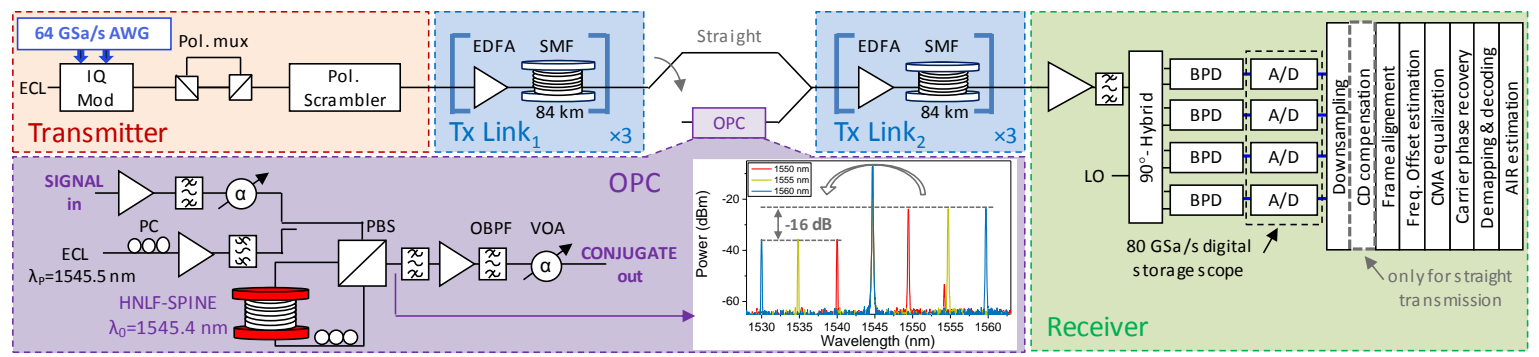

Fig. 1: Experimental setup for the comparison of straight and the OPC-based transmission. Inset shows the optical spectra at the output of the PBS, highlighting the three wavelength shifts considered: $10 \mathrm{~nm}, 20 \mathrm{~nm}, 30 \mathrm{~nm}$. 

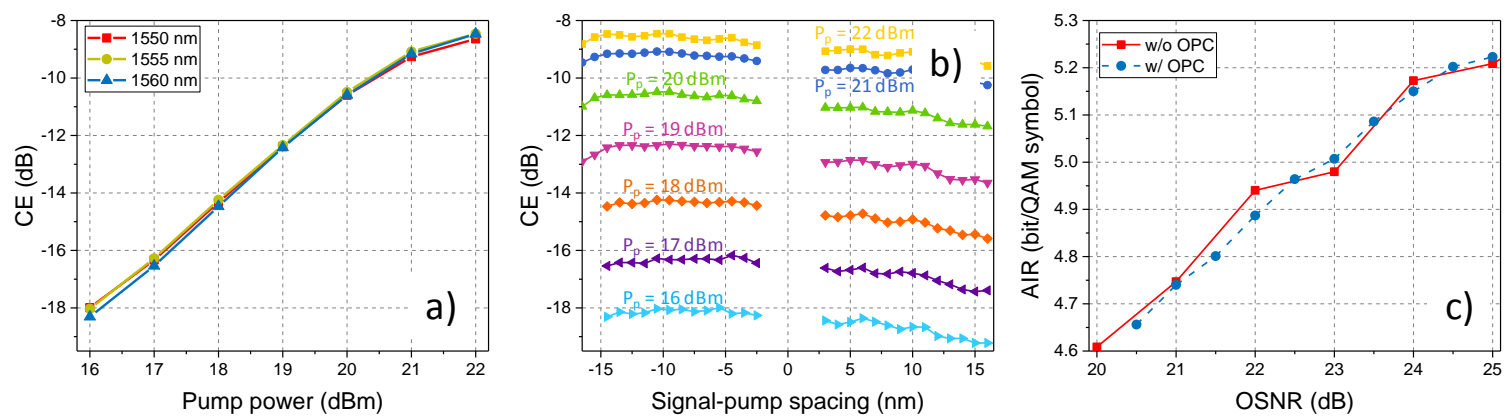

Fig. 2: a) Conversion efficiency (CE) as a function of the pump power (in straight HNLF configuration) for the three signal wavelengths considered; b) $\mathrm{CE}$ as a function of the signal-pump spacing for different pump power levels, yelding the conversion bandwidth; c) Achievable information rate (AIR) as a function of the signal OSNR in back-to-back configuration without and with polarization-independent OPC for 16-GBd 256-QAM.

has already been reported to provide improvements also for such asymmetric links ${ }^{5}$. After transmission, the signal is received in an 80-GSa/s pre-amplified coherent receiver (33$\mathrm{GHz}$ analog bandwidth) with a $10-\mathrm{kHz}$ linewidth ECL as local oscillator (LO). Offline digital signal processing (DSP) is then performed consisting of low-pass filtering, chromatic dispersion (CD) compensation (in straight configuration only), frequency offset estimation, pilot-based time domain equalization, and carrier phase recovery as in [9]. The metric chosen to evaluate the performance is the achievable information rate (AIR), estimated by using the auxiliary probability distribution derived from the phase recovery algorithm as discussed in [9]. The AIR is calculated over more than $10^{6}$ symbols to guarantee a reliable estimation and it is used to compare straight transmission, where the two links of 3 fiber spans are connected directly, with the OPC-based transmission, where the OPC operation is performed between the two links. In order to evaluate the dependence of the improvement provided by OPC on the wavelength shift, the signal wavelength has been varied by placing the signal at $1550 \mathrm{~nm}, 1555 \mathrm{~nm}$ and $1560 \mathrm{~nm}$, corresponding to signal-conjugate wavelength shifts of $10 \mathrm{~nm}, 20 \mathrm{~nm}$ and $30 \mathrm{~nm}$, respectively (pump wavelength of $1545.5 \mathrm{~nm}$ ).

\section{OPC stage characterization}

The OPC stage is based on single-pump FWM using a $500-\mathrm{m}$ highly nonlinear fiber (HNLF-SPINE) as the nonlinear medium. The FWM performance is shown in Fig. 2 (a)-(b) under continuous wave $(\mathrm{CW})$ operation with the pump placed at $1545.5 \mathrm{~nm}$. The conversion efficiency (CE) scales quadratically with the pump power $\left(P_{p}\right)$ up to $P_{p}=21 \mathrm{dBm}$ (Brillouin scattering threshold). Identical CE levels are achievable for the three signal wavelengths considered (Fig. 2(a)) leading to equivalent optical signal to noise ratio (OSNR) for the three conjugated copies. The full conversion bandwidth is shown in Fig. 2(b) achieving an operational bandwidth for the OPC stage covering most of the C-band with less than $2 \mathrm{~dB}$ of $\mathrm{CE}$ variation. To provide polarization-independent OPC operation, a polarization-diversity loop ${ }^{2}$ is used (see Fig. 1, lower left) with a polarizationdependence below $0.4 \mathrm{~dB}$. The pump power per propagation direction is $17 \mathrm{dBm}$ resulting in a CE of $-16 \mathrm{~dB}$. The spectra at the output of the diversity loop are shown in the inset of Fig. 1. The input signal power into the OPC has been optimized (in terms of AIR) to $-2 \mathrm{dBm}$, and kept constant by using a variable optical attenuator (VOA). At the output of the polarization beam splitter (PBS), two OBPFs with an EDFA in between and a VOA are used to suppress signal and pump and to amplify the conjugated copy such that the input power to the first EDFA of Link 2 is the same as in straight transmission. Low-penalty operation of the OPC stage is verified by back-to-back measurements with and without OPC performing OSNR loading at the receiver input. The estimated AIRs as functions of the signal OSNR are shown in Fig. 2(c) for 16-GBd 256-QAM, confirming that no signal degradation is introduced by the OPC stage.

\section{Nonlinearity compensation results}

In order to evaluate the improvement provided by nonlinearity compensation through OPC, the launched power into each span has been varied and the AIR has been measured at the receiver for the three signal wavelengths. The comparison between straight and OPC-based transmission is shown in Fig. 3(a) and (b) for 10-GBd 64-QAM and 10-GBd 256-QAM, respectively. In the linear transmission regime, straight and OPC-based transmissions show similar performance. The CE is sufficient to ensure that the OSNR of the conjugate signal is determined by the OSNR of the original signal confirming that the OPC does not degrade the signal. In the nonlinear regime, the nonlinearity compensation provided by OPC is clearly seen for both modulation formats. OPC enables an increase in the optimum launched power, from $0 \mathrm{dBm}$ to $+3 \mathrm{dBm}$, as well as a higher received AIR. More importantly, the three signal 

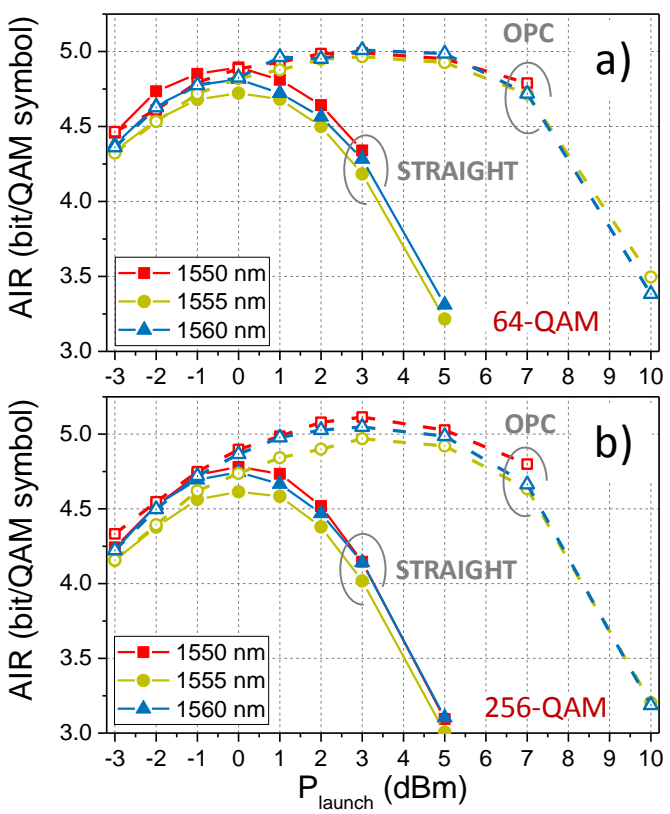

Fig. 3: Estimated AIR as a function of the launched power for straight and OPC-based transmission: 10-GBd 64-QAM (a) and 256-QAM (b) and the three signal wavelengths under investigation.

wavelengths show nearly identical improvements. The slightly lower AIR for the 1555-nm signal (both with and without OPC) is due to a minor wavelength dependence of the transmitter setup already noticeable in the linear regime. The difference in wavelength shift between the three configurations corresponds to an accumulated dispersion mismatch of approx. $215 \mathrm{ps} / \mathrm{nm}, 430 \mathrm{ps} / \mathrm{nm}$ and $646 \mathrm{ps} / \mathrm{nm}$ compared to a wavelength-shift free OPC. Note that a 30-nm wavelength shift is close to the maximum shift produced by an OPC stage covering the full C-band. The overall AIR improvement, defined as the difference in AIR between straight and OPCbased transmission at the optimum launched power, for the three signal wavelengths and all four pairs of modulation format and baudrate under investigation, are reported in Fig. 4. For all these cases, no notable wavelength dependent improvement variations are shown. The minor differences between signal wavelengths fall within the precision of the measurement of

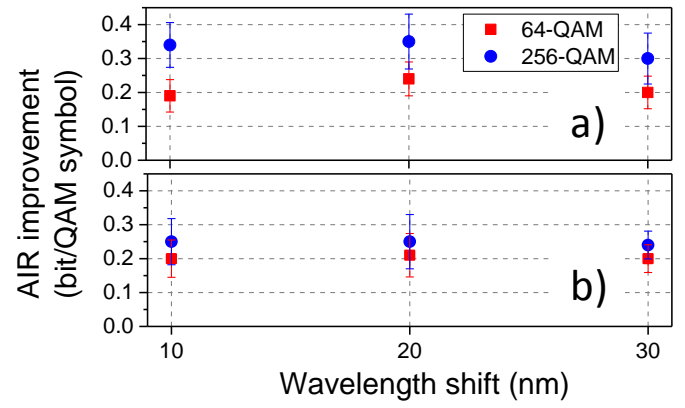

Fig. 4: AIR improvement as a function of the wavelength shift for (a) $10 \mathrm{GBd}$ and (b) $16 \mathrm{GBd}$ signals. approx. \pm 0.05 bit/QAM symbol (see error bars in Fig. 4).

\section{Conclusions}

We have investigated the impact of wavelength shift between a signal and its conjugated copy on the improvement provided by OPC in a $500-\mathrm{km}$ dispersion-uncompensated link with lumped EDFA amplification. Three signal wavelengths, $1550 \mathrm{~nm}, \quad 1555 \mathrm{~nm}$ and $1560 \mathrm{~nm}$, are considered, corresponding to a signal-conjugate wavelength shift of $10 \mathrm{~nm}, 20 \mathrm{~nm}$ and $30 \mathrm{~nm}$, respectively. AIR improvements between 0.2and $0.3 \mathrm{bit} / \mathrm{QAM}$ symbol provided by OPC are reported for 64-QAM and 256-QAM at $10 \mathrm{GBd}$ and $16 \mathrm{GBd}$, with no impact of the wavelength shift on the improvement. We believe that this constitutes an important confirmation of the great potential of OPC for nonlinearity compensation in practical communication links.

\section{Acknowledgements}

This work is supported by CoE SPOC, ref. DNRF123 and DFF-4005-00558 project NANO-SPECs.

\section{References}

[1] S. Watanabe, and M. Shirasaki, "Exact compensation for both chromatic dispersion and Kerr effect in a transmission fiber using optical phase conjugation," J. Lightwave Technol., Vol. 14, no. 3, p. 243 (1996).

[2] I. Sackey et al., "Kerr nonlinearity mitigation: mid-link spectral inversion versus digital backpropagation in 5×28-GBd PDM 16-QAM signal transmission," J. Lightwave Technol., Vol. 33, no. 3, p. 1827 (2014).

[3] P. Minzioni, "Nonlinearity compensation in a fiber-optic link by optical phase conjugation," Fiber and Integrated Optics, Vol. 28, no. 3, p. 179 (2009).

[4] M.E. McCarthy, et al., "PMD tolerant nonlinear compensation using in-line phase conjugation," Opt. Express, Vol. 24, no. 4, p. 3385 (2016).

[5] K. Solis-Trapala. et al., "Doubled transmission reach for DP-64QAM signal over field-deployed legacy fiber systems enabled by MSSI," Proc. ECOC, Mo.3.6.2, Valencia (2015).

[6] I. Sackey et al., "Novel wavelength-shift-free optical phase conjugator used for fiber nonlinearity mitigation in 200-Gb/s PDM-16QAM transmission," Proc. OFC, Th3J.1, Los Angeles (2017).

[7] K. Inoue, "Spectral inversion with no wavelength shift based on FWM with orthogonal pump beams," Opt. Lett. Vol. 22, no. 23, p. 1772 (1997)

[8] H.C. Lim et al., "Polarization-independent, wavelengthshift-free optical phase conjugator using a nonlinear fiber Sagnac interferometer", Photon. Technol. Lett., Vol. 11, no. 5, p. 578 (1999)

[9] M.P. Yankov, et al., "Constellation shaping for WDM systems using 256QAM/1024QAM with probabilistic optimization," J. Lightwave Technol., Vol. 32, no. 22, p. 5146 (2016). 